\title{
AJUSTAMENTO DIÁDICO E CONJUGALIDADE: AVALIAÇÃO DO BEM-ESTAR NO CASAMENTO
}

\author{
DYADIC ADJUSTMENT AND MARITAL RELATIONSHIP: EVALUATION OF \\ WELL-BEING IN MARRIAGE
}

\author{
Fabio Scorsolini-Comin' ${ }^{1}$, Manoel Antônio dos Santos²
}

\begin{abstract}
RESUMO:
Este estudo teve por objetivo discutir a utilização da Escala de Ajustamento Diádico (EAD) para a avaliação do construto conjugalidade, a partir da investigação das correlações entre as suas dimensões: coesão, consenso, satisfação e expressão do afeto. A escala foi aplicada em uma amostra de conveniência e não-probabilística de 106 pessoas casadas e coabitando há 16,11士11,35 anos. Os dados obtidos evidenciaram que todos os domínios da escala correlacionaram-se moderadamente entre si, embora a análise multivariada stepwise aponte que apenas a coesão diádica esteve diretamente associada ao consenso diádico e à expressão do afeto. Tal achado confirma a adequação da escala para a mensuração não apenas do ajustamento diádico, mas também da conjugalidade. Sugere-se a possibilidade de utilização combinada com outros instrumentos associados, que avaliem dimensões individuais de satisfação com o cônjuge.
\end{abstract}

Palavras-chave: casamento; estado conjugal; avaliação; escalas.

\begin{abstract}
:
This study aimed to discuss the employ of the Dyadic Adjustment Scale (DAS) to the evaluation of marital relationship, based on the investigation of correlations between the dimensions: cohesion, consensus, satisfaction and expression of affection. The scale was administered to a convenience sample and non-probabilistic 106 people married for $16.11 \pm 11.35$ years. Data show that all areas of the scale were moderately correlated with one another, although the stepwise multivariate analysis indicated that only dyadic cohesion was directly associated with the dyadic consensus and expression of affection. This finding confirms the adequacy of the scale to measure not only of dyadic adjustment, but also of marital relationship. It was suggested the possibility of using other instruments related to assess individual dimensions of satisfaction with the spouse.
\end{abstract}

Key words: marriage; conjugal status; evaluation; scales.

\section{INTRODUÇÃO}

A vida conjugal tem sido alvo de pesquisas em diferentes áreas do conhecimento, como Psicologia, Ciências Sociais, Antropologia, e também do campo da saúde, no qual destacamos a Saúde Coletiva e a Saúde da Família. Nessas áreas tem-se discutido a supervalorização de uma vida saudável $^{1-3}$, que implica, entre outros fatores, em desfrutar de uma vida conjugal harmoniosa com impac- tos não apenas na vida do casal como também na criação dos filhos e no desenvolvimento da família ${ }^{4-5}$. Assim, a assunção da conjugalidade tem sido relevada como uma questão de saúde para o casal e também para a família, sendo apontada como fator de proteção para doenças orgânicas e transtornos mentais, e fonte de apoio social importante frente a eventos estressores. Alguns estudos ${ }^{6-7}$ retratam a conjugalidade dos pais e as formas de transmissão de valores e práticas através das ge-

1 Professor Adjunto do Departamento de Psicologia da Universidade Federal do Triângulo Mineiro. E-mail: scorsolini_usp@yahoo.com.br

2 Professor em Psicologia da Faculdade de Filosofia, Ciências e Letras de Ribeirão Preto da Universidade de São Paulo. E-mail: masantos@ffclrp.usp.br

Corresponding author: scorsolini_usp@yahoo.com.br

Suggested citation: Scorsolini-Comin F, Dos Santos MA. Dyadic adjustment and marital relationship: evaluation of well-being in marriage. J. Hum. Growth Dev. 2012; 22(3): 367-372

Manuscript submitted Apr 28 2012, accepted for publication Aug 102012. 
rações como possíveis fatores que contribuiriam não apenas para o bem-estar dos filhos, mas também para a compreensão de questões complexas que estariam na raiz de problemas psicossociais, como o comportamento agressivo e a violência contra a mulher. Ao considerar essas implicações, compreender a dinâmica das relações familiares, nas quais se incluem as vivências afetivas conjugais, pode contribuir para o planejamento de estratégias de prevenção e de promoção da saúde ${ }^{8}$.

A conjugalidade ou identidade conjugal pode ser definida como o entrelaçamento das individualidades dos cônjuges, que dá origem à construção de uma identidade do casal, um espaço intersubjetivo que é continuamente transformado por ambos a partir da vivência conjugal ${ }^{9}$. Ainda que seja uma noção eminentemente psicanalítica, na atualidade tem sido investigada também a partir de instrumentos de mensuração, como forma de se apreender o fenômeno em sua intersecção com outras dimensões, como a de bem-estar psicológi$\mathrm{CO}^{10}$, diretamente ligado às estratégias de promoção da saúde ${ }^{6}$ na família.

Nesse contexto, alguns instrumentos têm sido desenvolvidos e testados, como é o caso do Questionário de Conjugalidade dos Pais ${ }^{11}$. Esse instrumento permite verificar o modo como a conjugalidade dos pais é organizada pela díade (casal) e como vem sendo elaborada e percebida pelos filhos. Avaliar a conjugalidade não significa apenas mensurar o grau de satisfação do casal com relação ao casamento. Estudos evidenciam que a avaliação da conjugalidade deve abarcar uma série de fatores, como a interação conjugal, a resolução de problemas conjugais, o significado da união para o casal, sua repercussão para a vida de cada um, as dificuldades enfrentadas no estabelecimento e manutenção da conjugalidade, entre outros aspectos relevantes ${ }^{12-17}$.

Na literatura científica internacional, a dimensão conjugal pode ser compreendida em termos do conceito de ajustamento conjugal ${ }^{18}$, uma noção relacionada ao ajustamento, comunicação, felicidade, integração e satisfação dos membros do casal $^{19}$. Para se estudar o ajustamento conjugal (ou ajustamento diádico) vem sendo largamente utilizada a Dyadic Adjustment Scale (DAS) ou Escala de Ajustamento Diádico (EAD), desenvolvida por Spanier ${ }^{18}$. Definir ajustamento conjugal como um processo tem diversas implicações, sendo a mais relevante o pressuposto de que um processo pode ser melhor investigado ao longo do tempo. A aplicação de estudos transversais na investigação do ajustamento tem algum valor, contudo, é evidente que um processo pode ser melhor observado mediante delineamentos longitudinais ${ }^{19}$.

A EAD é uma escala que já conta com adaptações para vários países e culturas ${ }^{16}$. No Brasil, foi utilizada em pesquisas que avaliam a satisfação conjugal ${ }^{19-21}$. No estudo original ${ }^{18}$, os índices de consistência interna encontrados evidenciaram alfa de Cronbach de 0,90 (consenso diádico), 0,94 (satis- fação diádica), 0,86 (coesão diádica) e 0,73 (expressão do afeto). No estudo de validação da escala para o contexto brasileiro ${ }^{18}$, utilizando uma amostra de 542 pessoas casadas, os níveis de consistência interna encontrados para a DAS total e suas subescalas foram similares aos produzidos nos estudos anteriores ${ }^{22-23}$, ou seja, classificados em uma escala de razoáveis a ótimos.

A EAD teve sua primeira análise fatorial confirmatória realizada em 1982 , na qual foi encontrada uma solução para quatro fatores, que explicaria $94 \%$ da covariância entre os itens. O coeficiente alfa de Cronbach para a escala toda foi de 0,91. A escala é constituída pelas dimensões: (a) Consenso diádico: avalia a percepção do nível de concordância do casal sobre uma variedade de questões básicas da relação, tais como: financeiras, lazer, religiosas, amizades, convencionalidade, filosofia de vida, entre outras; (b) Satisfação diádica: mede a percepção das questões relativas à discussão do divórcio, à saída de casa após briga, ao arrependimento com o casamento, à implicância mútua, ao estar bem, à confiança no cônjuge, entre outros ${ }^{19}$; (c) Coesão diádica: examina o senso de compartilhamento emocional do casal; (d) Expressão diádica de afeto: mede a percepção da concordância dos cônjuges sobre as demonstrações de afeto, as relações sexuais, a falta de amor e as recusas ao sexo ${ }^{19}$.

Ao considerar as dimensões constituintes da escala, o consenso diádico se refere ao compartiIhamento de ideias e perspectivas, o que é apontado como uma dimensão importante no casamento. Refere-se, ainda, à concordância do casal a respeito da conduta assumida frente a valores e normas sociais, organização das carreiras e tarefas domésticas, entre outros aspectos da vida conjugal que remetem à diversidade de condições de adaptação a que a pessoa pode ser exposta em um casamento ${ }^{18-19}$. O consenso abrange questões relacionadas à família, convenções sociais, formas de lidar com a família de origem, objetivos, metas e valores considerados importantes, quantidade de tempo que o casal passa junto, decisões relativas à carreira, o que implica em certo nível de concordância para que não se produza elevado nível de tensão e insatisfação no casamento.

A dimensão satisfação com a díade refere-se à percepção direta da satisfação conjugal, isto é, como cada cônjuge percebe seu casamento e também em relação à presença de alguns fatores ou comportamentos na vida conjugal que a tornam satisfatória ou não satisfatória ${ }^{17}$. Essa avaliação e percepção se dão, atualmente, de forma conflituosa, principalmente devido à fase de transição e ao privilégio do individual sobre o relacional e o coletivo. Por outro lado, essa percepção é amplificada pelo contínuo e crescente processo de equidade entre homens e mulheres - ao menos no plano manifesto dos discursos. Desse modo, ainda que tal avaliação contemple uma maior possibilidade de reflexão e de questionamentos acerca do casamento, 
dos papéis do cônjuge e de como cada um percebe e vivencia o relacionamento conjugal na contemporaneidade, observa-se que este domínio se correlaciona de modo significativo com todos os domínios da satisfação conjugal, o que pode ser devido à consonância existente entre as escalas e à própria definição dos construtos.

Em relação ao domínio da conjugalidade denominado coesão, este é compreendido como um sentimento ou vivência de união e integração entre os cônjuges. Engloba questões como o envolvimento em atividades extrafamiliares juntos, frequência de troca de ideias estimulantes sobre algum objeto, frequência com que o casal trabalha junto em algum projeto. Quando há o enfraquecimento da coesão, isso pode se dever tanto a fatores externos (compromissos e jornadas de trabalho ou planejamento de carreira), quanto a fatores internos ${ }^{17-19}$. A coesão se refere à proximidade e ao sentimento de conexão e intimidade percebidos pelo casal, havendo um compromisso partilhado com a relação e com sua continuidade, que resulta em um sentimento de preservação do relacionamento e do vínculo, de forma a minimizar as influências de outros sobre a relação conjugal. Em casamentos mais tradicionais a mulher ainda é a grande responsável pela manutenção da coesão do casal, pois ela parece se adaptar mais ao modelo do marido e suas necessidades subjetivas, do que o contrário ${ }^{17}$. Por último, dimensão conhecida como expressão do afeto é definida como a percepção subjetiva acerca da concordância ou discordância de um casal em questões relativas à forma e frequência de demonstrações de carinho, afeto e desejo sexual ${ }^{17}$.

$\mathrm{Na}$ literatura científica, os fatores da EAD (coesão, consenso, satisfação diádica e expressão do afeto) têm sido predominantemente abordados de modo individual, em termos de suas propriedades psicométricas ou apenas como componentes de medida geral que avalia o ajustamento entre o casal. Frente a essas considerações, o objetivo deste estudo é discutir a utilização da Escala de Ajustamento Diádico (EAD) para a avaliação do construto conjugalidade, a partir da investigação das correlações entre as dimensões que constituem o instrumento.

\section{MÉTODO}

Tipo de estudo: Trata-se de um estudo de caráter descritivo, transversal e correlacional.

Participantes: Do ponto de vista do perfil sociodemográfico, a amostra foi composta por 106 participantes (membros de 53 casais heterossexuais), legalmente casados havia, no mínimo, um ano, com ou sem filhos. A média em anos de casamento foi de $16,11 \pm 11,35$. No que concerne à idade dos participantes, a idade média foi de $42 \pm 11$ anos. A média de idade dos homens foi de 43,4 anos, ao passo que a das mulheres foi de 40,7 . Em relação ao número de filhos dos casais participantes, a média foi de 1,49 filhos para cada participante, com desvio padrão de 1,22. Em termos da classificação socioeconômica, a partir do critério Abipeme, a maioria dos participantes pertencia à classe B $(60,37 \%)$. Atrelada à classificação do status socioeconômico, a renda per capita foi de 5,03 salários, com desvio padrão de 3,63. Em relação ao grau de instrução dos participantes, a maioria $(66,98 \%)$ possuía nível superior, o que se observou tanto entre homens quanto entre mulheres.

Em relação aos critérios de inclusão/exclusão, não se restringiu duração máxima para os casamentos, idade, ocupação, quantidade de filhos ou classificação socioeconômica. Foram definidos como critérios de inclusão: estar consensualmente casado há, no mínimo, um ano; não apresentar indícios de comprometimento cognitivo ou comportamental; não estar em processo de separação conjugal. Neste estudo não foram estudadas as correlações intradíades (grau de consenso entre os membros do par), mas sim as respostas dadas por pessoas casadas, de modo independente.

Coleta dos dados: Os participantes foram selecionados pela técnica da bola de neve, em que novos participantes foram indicados pelos próprios respondentes, a partir dos contatos do pesquisador. Após serem esclarecidos sobre a pesquisa, os participantes assinaram o Termo de Consentimento Livre e Esclarecido e preencheram os questionários e escalas. A aplicação da bateria de instrumentos foi feita individualmente com cada participante, ou seja, mesmo se tratando de casais, cada membro respondeu de modo independente (sem a presença do cônjuge). Outro cuidado para assegurar a aplicação independente foi de que os respondentes não tivessem acesso às respostas do parceiro (acesso aos instrumentos respondidos e nem conversa após a aplicação, por exemplo), o que poderia influenciar nas respostas. Assim, a aplicação com cada cônjuge foi realizada de modo sequenciado e independente. Este estudo foi aprovado pelo Comitê de Ética em Pesquisa da Faculdade de Filosofia, Ciências e Letras de Ribeirão Preto, Universidade de São Paulo (Processo no 349/2007 2007.1.2016.59.0).

\section{Instrumentos:}

(a) Questionário Sociodemográfico: desenvolvido pelos autores deste estudo para identificação do participante e de suas condições socioeconômica e demográfica.

(b) Escala Abipeme de Classificação Socioeconômica: O critério Abipeme ${ }^{24}$ é uma escala ou classificação socioeconômica aferida por intermédio da atribuição de pesos a um conjunto de itens de conforto doméstico, além do nível de escolaridade do chefe de família.

(c) Escala de Ajustamento Diádico - EAD: Escala de origem norte-americana ${ }^{18}$ desenvolvida para a avaliação da percepção de casais acerca de seus relacionamentos 
afetivos. É uma escala mundialmente conhecida, com adaptações para vários países e culturas.

Análise dos dados: Os dados obtidos com a aplicação dos instrumentos foram transpostos para o Software SAS 9.2 e categorizados a partir de números de identificação, por casal, por sexo e, consequentemente, por participante. Foram calculadas as correlações entre as variáveis contínuas dos seguintes domínios: (a) consenso; (b) satisfação diádica; (c) coesão; (d) expressão do afeto (a, b, c e d são domínios de ajustamento diádico, pela DAS); (e) idade; e (f) classificação socioeconômica. A força da grandeza do coeficiente de correlação foi avaliada conforme procedimento proposto por Zou, Tuncali e Silverman ${ }^{25}$. O nível de significância adotado foi de $p d^{\prime \prime} 0,05$. Depois da definição das correlações, estabeleceu-se a relação dos escores totais dos diversos domínios a partir de um modelo linear múltiplo em que todas as variáveis foram consideradas a princípio. Para estabelecer o modelo final, todas as variáveis ( $a, b, c, d, e, f)$ foram submetidas ao método de seleção de variáveis stepwise, no qual permaneceram no modelo aquelas que apresentaram maior evidência de significância do ponto de vista multivariado. A adoção deste método permitiu filtrar, dentre todas as variáveis elencadas, as que tinham maior poder de predição, uma vez que se trata de uma ferramenta de análise adequada para examinar e entender todos os tipos de relacionamentos interdependentes ${ }^{26}$.

\section{RESULTADOS}

Correlacionando-se os domínios de conjugalidade (consenso, coesão, satisfação e expressão do afeto) entre si, observou-se que todos estavam correlacionados ( $p<0,05)$, o que corrobora os estudos que remontam à criação da $E A D^{18}$ e confirma a principal hipótese do presente estudo, que remete à consistência interna do construto em uma amostra composta apenas por pessoas casadas. É necessário destacar que no estudo anterior de validação para o contexto brasileiro ${ }^{21}$ foram incluídas pessoas que estavam em algum tipo de relacionamento amoroso heterossexual (casamento, namoro, relação extraconjugal) e que não necessariamente coabitavam.

No entanto, pela análise de regressão múltipla stepwise, quando se analisa cada uma das variáveis de conjugalidade em relação às demais do mesmo domínio, observou-se que não havia uma correlação significativa, a não ser o consenso, diretamente relacionado à coesão diádica, o que pode ser visualizado na Figura 1. Há que se destacar que os resultados (correlações) foram organizados por sexo, embora essa não seja uma variável elencada para análise no presente estudo.

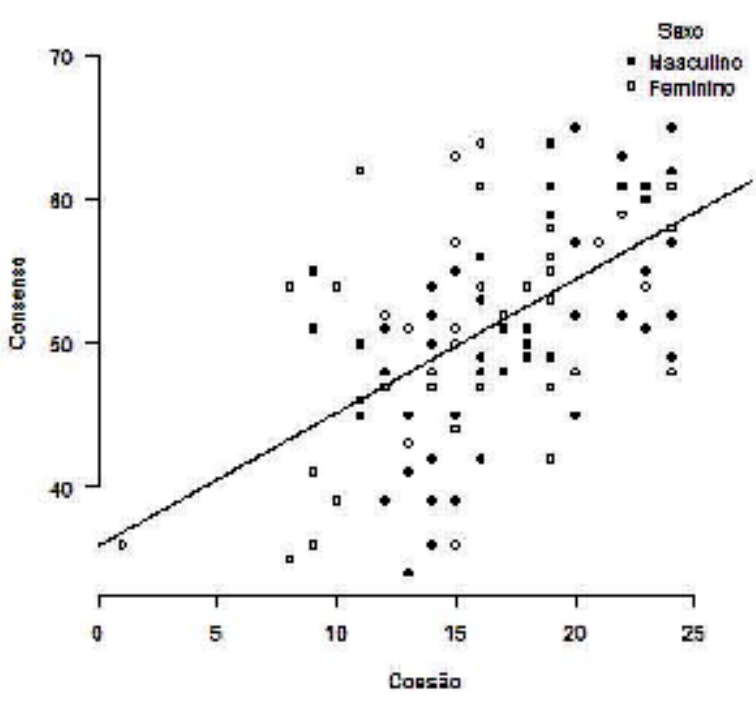

Figura 1: Correlação entre consenso e coesão diádica em participantes de ambos os sexos $(N=106)$

A partir da amostra de 106 pessoas casadas, verificou-se que o consenso está negativamente correlacionado com a satisfação (rho $=-0,25$; $\mathrm{p}<0,05$ ) (Figura 2 ) e positivamente correlacionado com a coesão (rho $=0,55 ; p<0,05$ ) e a expressão do afeto ( $r$ o $=0,45 ; p<0,05$ ). A partir da análise de regressão múltipla stepwise, verificou-se que o consenso está diretamente relacionado com a coesão diádica ${ }^{27-28}$

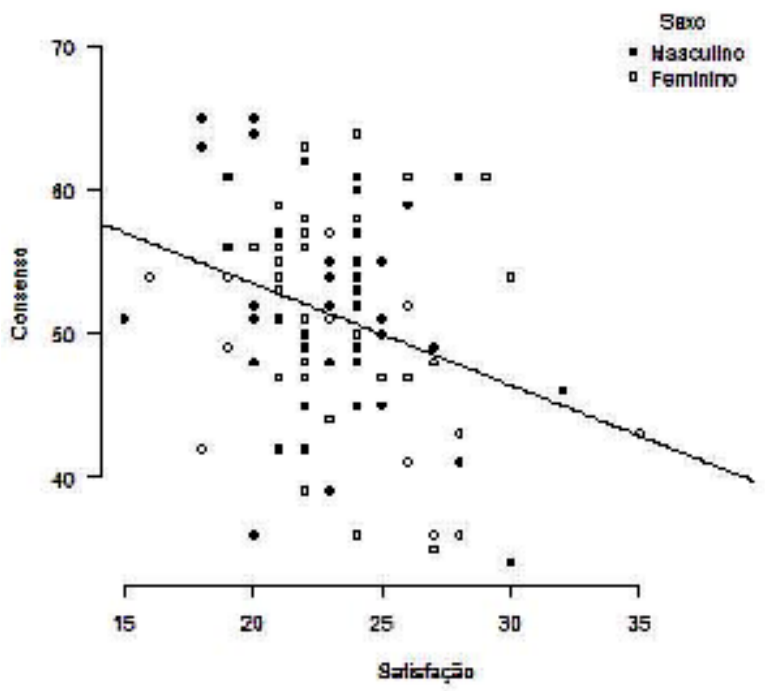

Figura 2: Correlação entre os domínios consenso diádico e satisfação diádica em participantes de ambos os sexos $(N=106)$.

Em termos da análise de regressão múltipla (Tabela 1), deve-se ponderar que o consenso está significativamente relacionado à coesão (ou seja, a coesão apresenta um papel preponderante sobre o consenso), mas o contrário não se mostrou verda- 
deiro. Assim, casais coesos tendem a ter maior nível de consenso, mas casais que experimentam grande consenso nem sempre são coesos.

Tabela 1: Resultados do modelo de regressão ajustado utilizando o método de seleção de variáveis stepwise $(N=106)$

\begin{tabular}{|c|c|c|c|}
\hline $\begin{array}{c}\text { Variável } \\
\text { Resposta* }\end{array}$ & $\begin{array}{c}\text { Variáveis } \\
\text { Explicativas }\end{array}$ & $\begin{array}{c}\text { Estimativa } \\
\text { do Parâmetro }\end{array}$ & p-valor \\
\hline Consenso diádico & Coesão & $\mathbf{0 , 4 5}$ & $<\mathbf{0 , 0 1}$ \\
& Satisfação diádica & $-0,35$ & 0,07 \\
Satisfação diádica & Idade & $-0,04$ & 0,10 \\
& Consenso & $-0,07$ & 0,13 \\
Coesão diádica & Consenso & 0,11 & 0,06 \\
& Expressão do afeto & 0,43 & 0,08 \\
Expressão do de casado & $-0,05$ & 0,10 \\
& Coesão & $\mathbf{0 , 0 8}$ & $\mathbf{0 , 0 4}$ \\
& Satisfação diádica & $-0,07$ & 0,13 \\
\hline
\end{tabular}

*A estimativa do parâmetro foi definida por meio do método de mínimos quadrados (Least Mean Square Method). Os resultados destacados em negrito são os mais significativos do ponto de vista estatístico.

O consenso não apresentou associações com as variáveis de idade ( $r$ o $=0,01 ; p=0,93$ ), tempo de relacionamento ( $r$ o $=0,10 ; p=0,47$ ); grau de instrução $(p=0,90)$; classificação socioeconômica $(p=0,31)$; e renda per capita (rho $=0,05$; $p=0,73)$.

A variável de conjugalidade denominada satisfação diádica está negativamente correlacionada apenas ao domínio de consenso. Não pode ser associada aos domínios de coesão (rho $=-0,13$; $\mathrm{p}=0,18$ ) e expressão do afeto (rho $=-0,14$; $p=0,16)$, o que vai em sentido oposto ao destacado pelo criador do instrumento, de que esses domínios são correlacionados significativamente ${ }^{18}$. Pela análise de regressão múltipla stepwise, a satisfação diádica parece não sofrer influências de quaisquer das dimensões da conjugalidade mensuradas pela EAD. A satisfação da díade não apresentou associações com a idade dos participantes ( $r$ o $=-0,15 ; p=0,12$ ), o tempo de relacionamento dos casais (rho $=-0,17 ; p=0,21$ ), o grau de instrução ( $p=0,13)$, a classificação socioeconômica $(p=0,11)$ e a renda per capita (rho $=0,08 ; p=0,59)$.

No presente estudo, a coesão se encontra positivamente correlacionada com a expressão do afeto ( $r$ ho $=0,47 ; p<0,01$ ). Esta correlação é considerada moderada ${ }^{25}$. Pela análise de regressão múltipla, a coesão não sofre influência significativa de quaisquer domínios da conjugalidade. Ainda de acordo com essa análise, a expressão do afeto seria direta e significativamente influenciada pela coesão diádica, o que reforça a hipótese da existência de um entrelaçamento entre os domínios da EAD.

\section{DISCUSSÃO}

Este estudo mostrou que os fatores da EAD estão correlacionados entre si, conforme observado em outros estudos ${ }^{21-22}$, embora não tenham sido encontradas correlações fortes entre todos esses fatores. As correlações mais significativas foram entre consenso e coesão e entre consenso e expressão do afeto. A satisfação diádica apresentouse negativamente correlacionada ao consenso. Não foi encontrado apoio na literatura para este achado. Pelo contrário, essa consideração é oposta ao que foi encontrado nos estudos conduzidos por investigadores vinculados ao grupo de pesquisa de Spanier ${ }^{18}$. A análise do tipo stepwise mostrou que a satisfação não é provocada por quaisquer dos domínios da conjugalidade, o que levanta a necessidade de elencar outros construtos ou variáveis que possam estar associados à satisfação diádica. Em estudo anterior foram encontradas associações entre a satisfação com os domínios do construto de bem-estar subjetivo ${ }^{16}$.

Outra consideração a ser destacada é que o consenso diádico ${ }^{17-19}$, por implicar em um nível de concordância entre o casal para que não se produza elevado nível de tensão e insatisfação no casamento, pode ser utilizado como um índice da qualidade do relacionamento conjugal. As pessoas casadas com maior consenso diádico tendem a ter uma vida conjugal mais harmoniosa e com menos conflitos, o que não implica em felicidade, mas permite pensar em estratégias de adaptação e flexibilidade frente às questões que possam promover discordâncias. Desse modo, a associação significativa entre consenso e coesão autoriza a postular que, quando há maior consenso (concordância, flexibilidade) entre os cônjuges, mais eles tendem a se manterem coesos (ou seja, unidos, podendo adotar posturas semelhantes e sem discrepância em relação ao casamento).

Em termos da análise de regressão múltipla stepwise, deve-se ponderar que o consenso manteve-se significativamente relacionado à coesão ou seja, a coesão apresenta um papel preponderante sobre o consenso. Porém, o contrário não se mostrou verdadeiro. Assim, pessoas casadas e com coesão no relacionamento tendem a ter maior nível de consenso, mas as casadas que experimentam grande consenso nem sempre são coesas. Pode-se depreender dessa relação que manifestar bom grau de concordância em relação aos diversos aspectos do casamento não implica, necessariamente, na união (coesão) entre os parceiros, nem mesmo na felicidade conjugal ${ }^{17-18}$.

Pelas correlações encontradas, sugere-se que as dimensões da conjugalidade avaliadas pelo instrumento em apreço possam ter uma abordagem independente, como se fossem quatro construtos da conjugalidade, por exemplo. Assim, os casais poderiam ser avaliados por meio apenas da satisfação diádica ou da expressão do afeto, a depender do objetivo do estudo. 
Outra possibilidade que pode ser considerada a partir dos dados obtidos no presente estudo é mensurar a conjugalidade a partir da integração de dois ou mais fatores que apresentem correlações mais significativas. Um exemplo disso seria avaliar o consenso e a coesão juntos, haja vista a forte associação existente entre esses fatores. Como a coesão diádica revelou ser um dos fatores mais diretamente associados ao consenso na amostra investigada, pondera-se a relevância de considerar ambos os fatores de modo integrado. Essa abordagem não desconsidera a análise dos fatores isolados, embora seja compreendida como um recurso auxiliar para compreender os fatores que, potencialmente, conduzem a relacionamentos satisfatórios.

Aponta-se, ainda, para o fato de que os domínios da conjugalidade estão mais centrados na avaliação da díade, do modo como cada membro do par um vivencia certos aspectos do casamento, ao passo que outros construtos, como a satisfação conjugal, levam em conta, além da interação conjugal, o modo como cada um avalia seu parceiro em termos de seus atributos emocionais e estruturais. Ou seja, cada cônjuge avalia esses aspectos presentes no outro, o que revela que o construto satisfação conjugal mensura não apenas domínios ligados à díade, mas também ao parceiro em termos individuais, ao passo que a conjugalidade (a partir do ajustamento diádico mensurado pela EAD) concentra-se no modo como o casal interage e constrói a sua identidade de casal, ou seja, sua conjugalidade.

Uma avaliação adequada da satisfação obtida com o relacionamento diádico deve vir acompanhada de bons índices de adaptação dos cônjuges, o que nos sugere que a conjugalidade deva ser investigada não apenas em termos da relação ou da percepção acerca da mesma, mas também dos

\section{REFERÊNCIAS}

1. Hernandes H, Horta ALM. Percepções de alunas de graduação em enfermagem sobre parcerias sorodiscordantes para o HIV/AIDS. Rev. Latino-Am. Enfermagem 2005; 13(4): 522529.

2. Foucault M. Naissance de la biopolitique: cours au College de France (1978-1979). Paris: Gallimard Seuil; 2004.

3. Reis RK, Gir E. Dificuldades enfrentadas pelos parceiros sorodiscordantes ao HIV na manutenção do sexo seguro. Rev. Latino-Am. Enfermagem 2005; 13(1): 32-37.

4. Menezes CC, Lopes RCS. A transição para o casamento em casais coabitantes e em casais não-coabitantes. Rev. Bras. Crescimento Desenvolv. Hum. 2007; 17(1): 52-63.

5. Bandeira TTA, Moura MLS, Vieira ML. Metas de socialização de pais e mães para seus filhos. Rev. Bras. Crescimento Desenvolv. Hum. 2009; 19(3): 445-456. aspectos expressos por cada um dos cônjuges. Sendo assim, avaliar a conjugalidade passa, inequivocamente, por uma avaliação do contexto no qual os cônjuges estão inseridos, de suas personalidades e de suas percepções acerca da própria vida, em termos individuais.

A partir dos resultados analisados, concluise que os casais com maior consenso diádico tendem a ter uma vida conjugal mais harmoniosa e menos permeada por conflitos, na medida em que conseguem se identificar com as necessidades de seus cônjuges. Isso não supõe, necessariamente, felicidade (entendida como satisfação conjugal), mas leva a pensar no uso de estratégias adaptativas de resolução de conflitos, baseadas na flexibilidade frente às questões que possam promover discordâncias. Tais estratégias poderiam ser articuladas de modo a promover bemestar na família e no domínio conjugal, minimizando os riscos de que as discordâncias conduzam tanto ao divórcio como a estratégias de resolução de conflitos disfuncionais, como a violência conjugal. A partir dessas evidências, uma possibilidade que se vislumbra para estudos futuros é a de investigar de que modo os aspectos desadaptativos da relação podem ser trabaIhados, de modo a favorecer que novas estratégias de resiliência conjugal possam ser utilizadas rumo à concordância do casal e o consequente incremento de satisfação, com vistas à promoção da saúde do casal.

Considerando os resultados apresentados, há evidências de que a EAD se constitui em um instrumento apropriado para a mensuração da conjugalidade, além de possibilitar abordagens complementares que, para além de fornecerem uma medida geral, destaquem as associações entre seus fatores como um aspecto relevante na compreensão do relacionamento conjugal.

6. Lamoglia CVA, Minayo MCS. Violência conjugal, um problema social e de saúde pública: estudo em uma delegacia do interior do Estado do Rio de Janeiro. Ciênc. saúde coletiva 2009; 14(2): 595-604.

7. Pesce R. Violência familiar e comportamento agressivo e transgressor na infância: uma revisão da literatura. Ciênc. saúde coletiva 2009; 14(2): 507-518.

8. Cabral MAA. Prevenção da violência conjugal contra a mulher. Ciênc. saúde coletiva 1999; 4(1): 183-191.

9. Féres-Carneiro T. Casamento contemporâneo: o difícil convívio da individualidade com a conjugalidade. Psicologia: Reflexão e Crítica 1998; 11(2): 379-394.

10. Scorsolini-Comin F, Santos MA. (2011). Relações entre bem-estar subjetivo e satisfação conjugal na abordagem da Psicologia Positiva. Psicologia: Reflexão e Crítica 2011; 24(4): 658-665. 
11. Ziviani C, Féres-Carneiro T, Magalhães AS, Bucher-Maluschke J. Avaliação da conjugalidade. In: Noronha APP, Santos AAA, Sisto FF (Orgs.). Facetas do fazer em avaliação psicológica. São Paulo: Vetor; 2006. p. 13-55

12. Dela Coleta MF. A medida da satisfação conjugal: Adaptação de uma escala. Psico 1989; 18(2): 90-112.

13. Dela Coleta MF. Locus de controle e satisfação conjugal. Psicologia: Teoria e Pesquisa 1992; 8(2): 243-252.

14. Pick de Weiss S, Palos AP. Desarrolo y validacion de la escala de satisfaccion marital. Psiquiatria 1988; 1: 9-20.

15. Norgren MBP, Souza RM, Kaslow F, Hammerschmidt H, Sharlin SA. Satisfação conjugal em casamentos de longa duração: uma construção possível. Estudos de Psicologia 2004; 9(3): 575-584.

16. Magagnin C, Kõrbes JM, Hernandez JAE, Cafruni $S$, Rodrigues MT, Zarpelon M. Da conjugalidade à parentalidade: gravidez, ajustamento e satisfação conjugal. Aletheia 2003; 17/18: 41-52.

17. Perlin GDB. Casamentos contemporâneos: um estudo sobre os impactos da interação famíliatrabalho na satisfação conjugal. [tese]. Brasília: Instituto de Psicologia da Universidade de Brasília; 2006.

18. Spanier GB. Measuring dyadic adjustment: new scales for assessing quality of marriage and similar dyads. Journal of Marriage and the Family 1976 ; 38(1): 15-28.

19. Hernandez JAE, Hutz CS. Gravidez do primeiro filho: papéis sexuais, ajustamento conjugal e emocional. Psicologia: Teoria e Pesquisa 2008; 24(2): 133-141.

20. Perlin G, Diniz G. Casais que trabalham e são felizes: mito ou realidade? Psicologia Clínica 2005; 17(2): 15-29.

21. Hernandez JAE. Avaliação estrutural da escala de ajustamento diádico. Psicologia em Estudo 2008; 13(3): 593-601.

22. Spanier GB, Thompson L. A confirmatory analysis of the Dyadic Adjustment Scale. Journal of Marriage and the Family $1982 ; 44(3)$ : 731-738.

23. Graham JM, Liu Y, Jeziorski JL. The Dyadic Adjustment Scale: a reliability generalization meta-analysis. Journal of Marriage and Family 2006; 68(3): 701-717.

24. Associação Brasileira dos Institutos de Pesquisa de Mercado - Abipeme. Critério de classificação socioeconômica - Brasil (CCSEB); 2008.

25. Zou KH, Tuncali K, Silverman SG. Correlation and simple linear regression. Radiology 2003; 227: 617-628.

26. Hair JFJ, Anderson RE, Tatham RL, Black WC. Multivariate data analysis with readings. $4^{\text {th }}$ ed. New Jersey: Prentice Hall; 1995.

27. Scorsolini-Comin F, Santos MA. Ajustamento diádico e satisfação conjugal: correlações entre os domínios de duas escalas de avaliação da conjugalidade. Psicologia: Reflexão e Crítica $2011 ; 24(3)$ : 439-447.

28. Scorsolini-Comin F, Santos MA. Satisfação com a vida e satisfação diádica: correlações entre construtos de bem-estar. PsicoUSF 2010; 15(2): 249-256. 